\title{
Fatigue limit evaluation of martensitic steels with thermal methods
}

\author{
by U. Galietti*, D. Palumbo*, R. De Finis* and F. Ancona*
}

* Politecnico di Bari, Dipartimento di Meccanica, Matematica e Management, Viale Japigia 182, 70126 Bari, Italy, galietti@poliba.it, d.palumbo@poliba.it, r.definis@libero.it, francesco.ancona@live.it.

\begin{abstract}
Fatigue behavior is a discriminating feature in choice of steels for extreme conditions such as high or low temperatures and corrosive environments. This is the case of martensitic steels considered in this work, ASTM A182 grade F6NM, and VIRGO 39. These kind of steels are characterized by temperature changes of material related to fatigue damage very low. The fatigue behavior of these steels was investigated with two thermal methods based on temperature surface monitoring and phase variation of thermoelastic signal (TPA method). Two procedures were developed to process thermographic data and to assess fatigue limit of materials.
\end{abstract}

\section{Introduction}

Thermal methods in this years are become a useful tool to study fatigue behaviour of materials. These methods allow to assess the fatigue limit of material reducing the experimental campaigns in terms of times and costs.

In literature different methods were developed considering temperature as parameter to describe the damage of material. In fact, during a fatigue test, the temperature variation between the maximum instantaneous temperature reached in the quasi-isothermal phase and the initial surface radiometric temperature on the specimen, increases when the stress is higher than the fatigue limit [1-6]. However, temperature depends on a variety of parameters such as stress amplitude, loading frequency, specimen geometry and above all environmental conditions that affect the heat exchange conditions between specimen and environment [7], [8].

A different approach is based on monitoring of heat sources that are generated during a fatigue test [7-9]. In the case that phase solid transformations don't occur during the test, two heat sources are generated: dissipative and thermoelastic sources. Dissipative sources are correlated with the damage of material and cause the temperature growth of specimen while thermoelastic sources are reversible and due to thermomechanical coupling phenomena.

Other methods are based on a specific data processing of recorded infrared sequences [10], [11]. In particular, in this case, the infrared signal was processed in time domain in order to obtain the information about the second order frequency of thermographic signal directly correlated to damage phenomena.

All these approaches can be used to estimate the fatigue limit of base material and welded joints [11-16]. However, for some materials, such as aluminium alloys, characterized by a high thermal conductivity coefficient and then a high thermal diffusivity, temperature changes related to fatigue damage are very low and so, it's very difficult to carry out an efficient damage monitoring. Another example concerns more complex mechanical components, such as welded joints, that are characterized by different fatigue failure mechanisms that involve the presence of very low energy thermal sources. Moreover, different thermal behaviours were observed for ductile and brittle material. In the last case, the fatigue damage is characterized by low strains that involving low temperature variations. In all these cases is required an high performance equipment and a more accurate setup.

In this work, the fatigue behaviour of martensitic steels was studied by means of thermographic techniques. A new approach was used based on TSA (Thermoelastic Stress Analysis) technique called TPA (Thermoelastic Phase Analysis). This approach was used to detect and monitoring eventual plastic behaviours of the material and also a procedure was proposed for fatigue limit assessment and damage monitoring.

TPA method uses the thermoelastic phase signal as parameter to describe the fatigue behaviour of material. Phase variations can occur in presence of heat generation due to the high gradient stress or local plasticity due to dissipative sources [13].

The proposed procedure based on the analysis of the evolution of signal phase variation over time during the same loading procedure used for temperature data analysis allows the reduction of problems related to temperature measurement [13].

Two martensitic steel were investigated: ASTM A182 grade F6NM and Virgo 39. Three specimens were tested for each material acquiring with two different thermocameras, thermographic and thermoelastic data. So, two different procedures based on thermographic and thermoelastic data were proposed and then two parameters were used to describe the fatigue damage of material.

The Stair-Case method on fifteen specimens was carried out on ASTM A182 in order to obtain a reference value for fatigue limit 


\section{Theory}

TSA provides a $S$ signal proportional to the peak-to-peak variation in temperature during the peak-to-peak variation of the sum of principal stress [16-18]. When adiabatic conditions occur, in case of linear elastic behaviour of homogeneous materials, the temperature variations and the stress are directly correlated through the follows equation [19]:

$$
\frac{\partial \theta}{\partial t}=-K T_{0} \frac{\partial \sigma_{I}}{\partial t}
$$

where, $\theta=T-T_{0}$ represents the temperature variations recorded during the fatigue test, $T_{0}$ is the equilibrium temperature field, $K$ is the thermoelastic constant [18] and $\sigma_{l}$ is the stress invariant.

The loss of adiabatic conditions occurs when is present heat transfer through the specimen or when an internal heat source arises due for example to damage phenomena. In this case other terms should be considered in eq. 1 and the stresses measurement would affected by errors. So, eq. 1 becomes:

$$
\frac{\partial \theta}{\partial t}=-K T_{0} \frac{\partial \sigma_{I}}{\partial t}+a \nabla^{2} \theta+\frac{d_{1}}{\rho C_{p}}
$$

with $a$ the thermal diffusivity, $\nabla^{2} \theta$ the temperature gradient, $C_{P}$ the specific heat at constant pressure, $\rho$ the mass density and $d_{1}$ the intrinsic dissipation; this latter occurs in the presence of plastic, viscoplastic or fatigue damage processing.

The usual TSA set-up involves a reference signal provided by a load cell or a strain gage on the specimen, that is used to filter the thermoelastic signal by means of a hardware lock-in amplifier unit or via software in post-processing.

Thermoelastic signal and reference signal are generally represented pixel by pixel as two vectors rotating at the same speed (same frequency) with constant phase shift. Hence, the thermoelastic signal is represented by its module ( $R$-image) proportional to temperature amplitude and then stress amplitude [16-18] and by phase angle with respect to reference signal (phase-image).

During a fatigue test a phase shift respect to reference signal occurs whenever the adiabatic conditions are lost. This can occurs when (considering eq. 1):

- $\quad$ is present the term $a \nabla^{2} \theta$ and it is not negligible respect to loading time variation $(\partial \sigma / \partial t$ term);

- $\quad$ are present dissipative heat sources $\left(d_{1} / \rho C_{P}\right)$ due to plasticity and other fatigue damage phenomena such as the arise of cracks and their subsequent growth.

So, phase signal can be used to monitor the damage of material during a fatigue test. The method that uses the phase as damage parameter is called TPA (Thermoelastic Phase analysis) and it was used also for the damage evaluation of welded joints [13].

The following paragraphs show how the phase shift represents a powerful tool for the evaluation of plastic zones, location of cracks as well as for the assessment of the fatigue limit of material.

\section{Experimental set-up}

\subsection{Materials}

Martensitic stainless steels have a high mechanical strength obtained by a quenching heat treatment but their corrosion resistance is limited. In fact the low alloy levels allow to obtain the complete transformation of austenite in martensitic structure. In particular, for the materials treated in this work, the cooling rates of quenching heat treatment are higher than other stainless steels in order to obtain an high strength of material [20].

The addition of Chromium (between $11.5 \%$ and $14 \%$ in weight for ASTM A182 and $16 \%$ in weight for VIRGO 39), allows to improve corrosion resistance by formation of the oxides, and it also permits to avoid the depleting of Chrome from lattice [20].

In table 1 are shown the information about the mechanical properties of analyzed materials. In particular are reported: the UTS (ultimate tensile strength) and the Yield stress (at $0.2 \%$ offset). 
Table 1. Mechanical properties of ASTM A182 and VIRGO 39

\begin{tabular}{|c|c|c|c|c|}
\hline Material & $\begin{array}{c}\text { Testing } \\
\text { Temperature } \\
{\left[{ }^{\circ} \mathbf{C}\right]}\end{array}$ & $\begin{array}{c}\text { Ultimate tensile } \\
\text { Strength [MPa] }\end{array}$ & $\begin{array}{c}\text { Young modulus } \\
{[\mathrm{MPa}]}\end{array}$ & $\begin{array}{c}\text { Yield stress (0,2\%) } \\
{[\mathrm{MPa}]}\end{array}$ \\
\hline ASTM A 182 grade F6NM & 20 & 766.8 & 198.5 & 615.7 \\
\hline VIRGO 39 & 20 & 911.6 & 194.6 & 632.5 \\
\hline
\end{tabular}

Three "dog bone" specimens for each material, sized according to ASTM E 466-96 [21], have been used for the tests. In figure 1 are reported the most important dimensions of specimen: nominal gauge length, nominal thickness and nominal width.

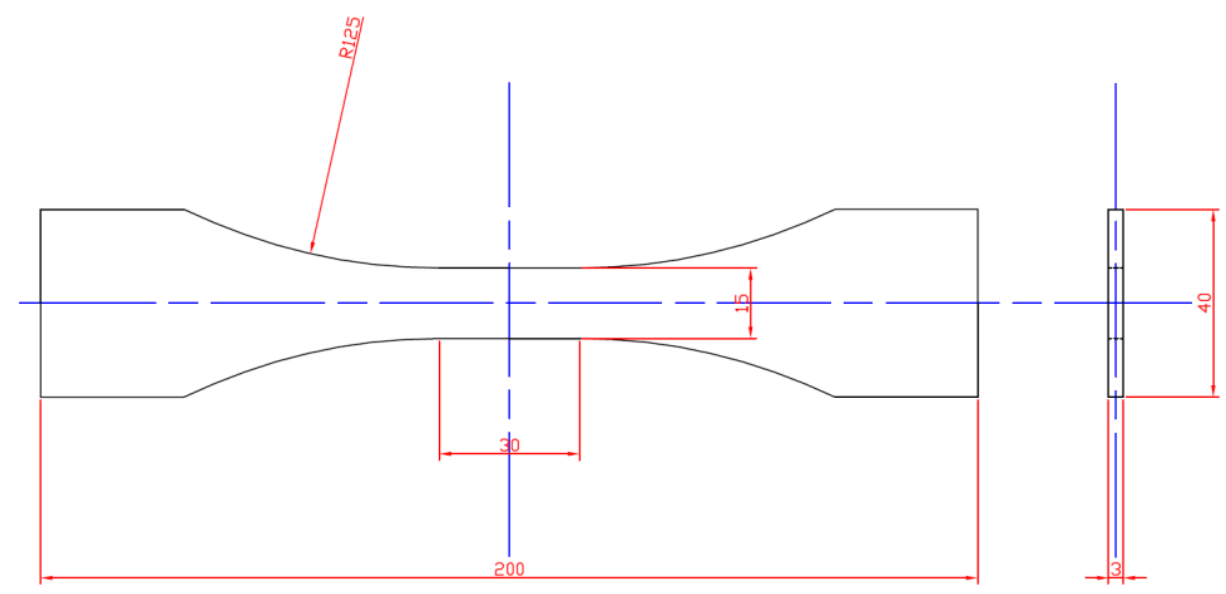

Fig. 1. Dimensions $(\mathrm{mm})$ and geometry of specimens

\subsection{Instrumentation and test procedures}

The instruments used for testing materials are: two infrared cameras, and a loading machine.

The IR camera X6540 SC manufactured by FLIR, it's capable to acquire thermographic sequences at elevated frame rate so, it results suited to carried out thermoelastic stress analysis tests. It is based on a indium-antimonide focal plane array detector that allows to acquire frames composed by $640 \times 512$ pixels. The full frame rate is about $125 \mathrm{~Hz}$ with a thermal sensitivity NETD $<20 \mathrm{mK}$.

A second IR camera FLIR A20 was used to obtain thermographic data. It is based on a microbolometric detector (160x140 pixels) with a thermal sensitivity NETD $<50 \mathrm{mK}$. The two IR cameras were placed on opposite side of specimen.

The tests were carried out with a MTS model 370 servo hydraulic fatigue machine with a $100 \mathrm{kN}$ capacity.

The "dog bone" specimens are sprayed with flat black spray in order to obtain high emissivity. The specimen was enclosed in a wood chamber to avoid heat reflections due to not considered external heat sources (figure 2).

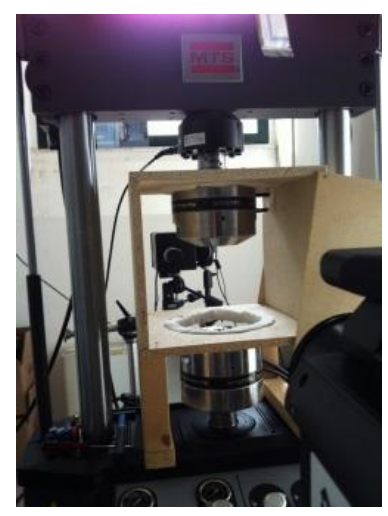

a

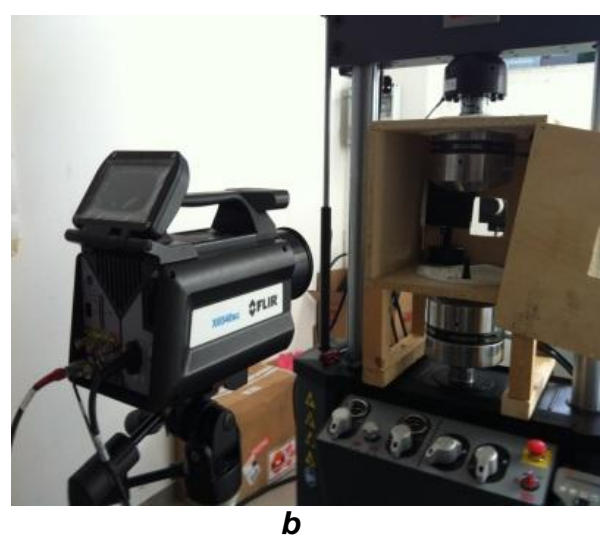

$\boldsymbol{b}$

Fig. 2. Wood chamber and IR cameras setup. 
chamber.

A painted unloaded aluminium plate is used as a black body to obtain a temperature reference inside the wood

Uniaxial tensile loading was applied (stress ratio $\mathrm{R}=0.5$ and frequency of $17 \mathrm{~Hz}$ ) for 20.000 cycles and then amplitude was increased as stated in table 2 and 3 up to the failure of material. At the end of each step loading machine was stopped and the load was incremented.

Thermographic sequences used for TSA were acquired with Flir X6540 Sc IR camera. In particular, three sequences were acquired for each load step respectively at 1000/8000/16000 cycles of loading machine. Each sequence was captured at $100 \mathrm{~Hz}$ for 10 seconds.

Thermographic data were acquired by Flir A20 and consist only in one sequence captured during the whole test until the failure.

Table 2. Test performed on VIRGO 39 (left) and ASTM A182 (right)

\begin{tabular}{|c|c|c|c|c|c|}
\hline STEP & $\boldsymbol{\Delta} \boldsymbol{\sigma} / \mathbf{2}$ & $\boldsymbol{\Delta} \boldsymbol{\sigma}$ & $\boldsymbol{\sigma}$ min & $\boldsymbol{\sigma}$ max & $\boldsymbol{\sigma}$ mean \\
\hline 1 & 25 & 50 & 50 & 100 & 75 \\
\hline 2 & 45 & 90 & 90 & 180 & 135 \\
\hline 3 & 65 & 130 & 130 & 260 & 195 \\
\hline 4 & 85 & 170 & 170 & 340 & 255 \\
\hline 5 & 105 & 210 & 210 & 420 & 315 \\
\hline 6 & 120 & 240 & 240 & 480 & 360 \\
\hline 7 & 135 & 270 & 270 & 540 & 405 \\
\hline 8 & 150 & 300 & 300 & 600 & 450 \\
\hline 9 & 165 & 330 & 330 & 660 & 495 \\
\hline 10 & 180 & 360 & 360 & 720 & 540 \\
\hline 11 & 190 & 380 & 380 & 760 & 570 \\
\hline 12 & 200 & 400 & 400 & 800 & 600 \\
\hline 13 & 208 & 415 & 415 & 830 & 623 \\
\hline 14 & 215 & 430 & 430 & 860 & 645 \\
\hline 15 & 223 & 445 & 445 & 890 & 668 \\
\hline 16 & 230 & 460 & 460 & 920 & 690 \\
\hline
\end{tabular}

\begin{tabular}{|c|c|c|c|c|c|}
\hline STEP & $\boldsymbol{\Delta} \boldsymbol{\sigma} / \mathbf{2}$ & $\boldsymbol{\Delta} \boldsymbol{\sigma}$ & $\boldsymbol{\sigma} \mathbf{m i n}$ & $\boldsymbol{\sigma}$ max & $\boldsymbol{\sigma}$ mean \\
\hline 1 & & & & & \\
\hline 2 & 35,00 & 70 & 70 & 140 & 105 \\
\hline 3 & 45,00 & 90 & 90 & 180 & 135 \\
\hline 4 & 52,50 & 105 & 105 & 210 & 158 \\
\hline 5 & 60,00 & 120 & 120 & 240 & 180 \\
\hline 6 & 67,50 & 135 & 135 & 270 & 203 \\
\hline 7 & 75,00 & 150 & 150 & 300 & 225 \\
\hline 8 & 82,50 & 165 & 165 & 330 & 248 \\
\hline 9 & 90,00 & 180 & 180 & 360 & 270 \\
\hline 10 & 97,50 & 195 & 195 & 390 & 293 \\
\hline 11 & 105,00 & 210 & 210 & 420 & 315 \\
\hline 12 & 112,50 & 225 & 225 & 450 & 338 \\
\hline 13 & 120,00 & 240 & 240 & 480 & 360 \\
\hline 14 & 127,50 & 255 & 255 & 510 & 383 \\
\hline 15 & 135,00 & 270 & 270 & 540 & 405 \\
\hline 16 & 142,50 & 285 & 285 & 570 & 428 \\
\hline 17 & 150,00 & 300 & 300 & 600 & 450 \\
\hline 18 & 157,50 & 315 & 315 & 630 & 473 \\
\hline 19 & 165,00 & 330 & 330 & 660 & 495 \\
\hline 20 & 172,50 & 345 & 345 & 690 & 518 \\
\hline 21 & 180,00 & 360 & 360 & 720 & 540 \\
\hline 22 & 187,50 & 375 & 375 & 750 & 563 \\
\hline 23 & 195,00 & 390 & 390 & 780 & 585 \\
\hline 24 & 202,50 & 405 & 405 & 810 & 608 \\
\hline 25 & 210,00 & 420 & 420 & 840 & 630 \\
\hline
\end{tabular}




\section{Description of IR data processing}

\subsection{Temperature data processing}

The temperature of specimen during fatigue test can be due to different heat sources (eq. 3) that could affect the measurement of the dissipative sources. In fact, the environmental temperature $T_{a m b}(t)$ and the heating of the loading machine grip $T_{I m}(x, y, t)$ contribute to the temperature growth of specimen [15].

$$
T(x, y, t)=f\left[T_{d}(x, y, t), T_{a m b}(t), T_{l m}(x, y, t)\right]
$$

In eq. 3, $x$ and $y$ coordinates, refer to a generic point on specimen gauge length.

Referring to (eq. 3) the only temperature contribution caused by damage in the specimen at a specific load is the term $T d(x, y, t)$. Therefore, it's necessary to eliminate the effects due to $T_{a m b}(t)$ and $T_{\operatorname{lm}}(x, y, t)$ that can compromise the correct measurement of dissipative source. As will be showed in the next paragraph a not accurate measurement of temperature could compromise the evaluation of fatigue limit of material.

In order to filter the noise component of temperature already described, it was adopted a new procedure of data processing. It was performed with Matlab ${ }^{\circ}$ considering only the gauge length of specimen as shown in figure 3 .

Figure 3 shows a thermographic image acquired at a given time during the loading step 19 (corresponding to $\Delta \sigma / 2=165 \mathrm{MPa}$ ) and related to specimen 1 of martensitic steel ASTM A182 grade F6NM.

In figure $3(\mathrm{a})$, the areas $A_{1}$ and $A_{2}$ refer, respectively, to the specimen gauge area, and black body area used for evaluation of room temperature $T_{a m b}(t)$. $T_{a m b}(t)$ represents the maximum value measured on black body and then on $A_{2}$ area. In order to eliminate instantaneous $T_{\text {amb }}(t)$ value, this one was subtracted pixel to pixel from $A_{1}$ area.

By plotting the temperature trend along a generic profile $p$ (figure 3 ), it is clear the effect of hot oil in the low cylinder of loading machine. This latter heats the specimen during the whole test and the heat provided is not constant but it growing during the test. In correspondence of dissipative phenomena a non symmetrical thermal profile appears figure $3(b-c)$. A straight line was used to interpolate the first and last point of $p$ profile. $A_{1}$ area is constituted by a number of $p$ profiles equal to number of pixels along $x$ axis so, subtracting the slope of straight line relative to each temperature profile of $A_{1}$ area, the effect of loading machine can be smoothed.

During a fatigue test the surface temperature at the beginning increases (first phase), then it remains constant (phase 2) and immediately before the failure it suddenly increases (phase $3-[5]$ ). Figure 4 shows the typical temperature trend that is obtained during a fatigue test carried out with a step loading procedure.

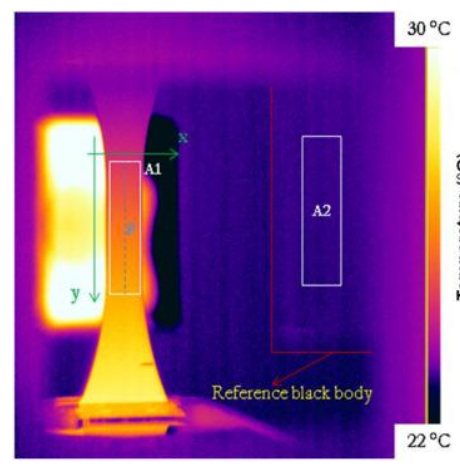

a

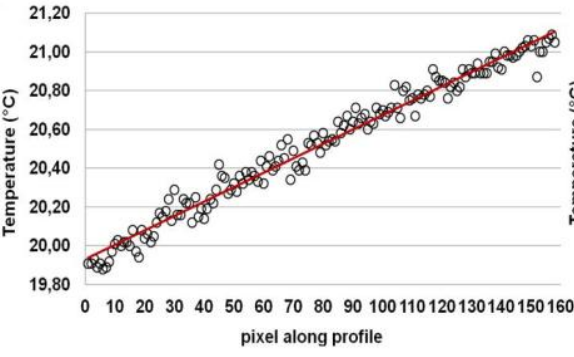

b

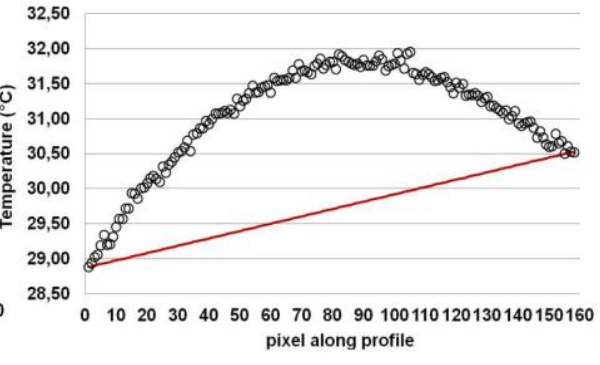

$c$

Fig. 3. a) Areas and thermal profile $p$ considered for analysis (ASTM A182, specimen 1): b) step 1, c) step 19.

In this case, after the smoothing procedure, the $\Delta T_{\max }$ were evaluated. These values are correlated to dissipative sources $\left(T_{d}(t)\right)$ and then to damaged phenomena.

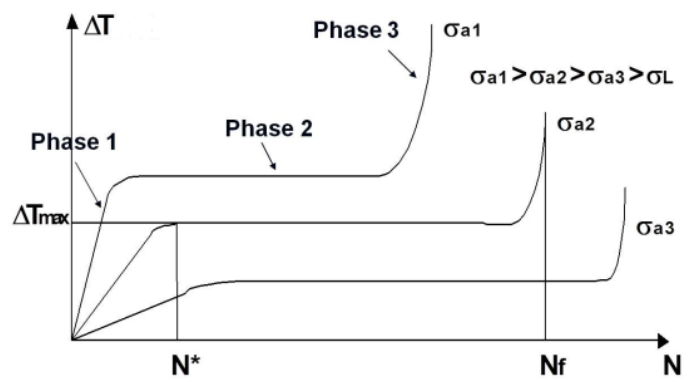

Fig. 4. Temperature trend during a fatigue test. 


\subsection{Thermoelastic data processing: TPA method}

As already described in the previous paragraphs, during fatigue tests and during each loading step, three thermal sequences were acquired respectively at 1000, 8000 and 16000 cycles. In order to obtain the information about the amplitude and phase of thermographic signal and then to perform the TSA, a suited algorithm was used to analyze the IR sequences.

For each pixel, a temperature model [10] was assumed to describes the experimental temperature over time measured during the fatigue test. The model is described by eq. (4):

$$
T_{m}(t)=a+b t+\Delta T_{1} \sin \left(\omega t+\varphi_{1}\right)+\Delta T_{2} \sin \left(2 \omega t+\varphi_{2}\right)
$$

where, $\Delta T_{1}, \varphi 1, \Delta T_{2}$ and $\varphi_{2}$ are the amplitude and phase of first and second Fourier component while the constants $a$ and $b$ were used to model the growth of temperature that occurs when are present damaged into material.

All the constants were obtained through a last square fit method imposing the model of eq. (4) to the thermographic signal of each pixel. The algorithm provides an image in form of matrix data for each parameters of eq. (4) (in total 6 images).

In this work only the phase signal and then the $\varphi_{1}$ constant will be take in account while, the remaining parameters will be discuss in future works.

During the data processing, the evaluation and subtraction of the phase mean value, allows the monitoring of phase variation for each step within the specimen.

Moreover, phase values were obtained by subtracting from each image a reference image taken during the first loading steps when there was no damage, in order to assess phase variations compared to a reference condition. This procedure allows to reduce the influence of external factors (such us painting or surface non homogeneity) on the phase variation reducing the standard deviation for the monitoring area not due to damage mechanism.

Finally, the maximum and the minimum value of the phase signal were evaluated and the $\Delta \varphi_{\max }=\varphi_{\max }-\varphi_{\min }$ was assessed. However, the $\varphi_{\max }$ and $\varphi_{\min }$ values were evaluated considering respectively the 98th and the 2 th percentile in order to avoid some outlier not removed by data processing procedure.

\section{Analysis and results.}

\subsection{Temperature analysis: Comparison between filtered and unfiltered data.}

In the previous paragraphs was exposed the procedure used in this work to determine the dissipative source. This procedure consist on the evaluation of steady state temperature $\Delta T_{\max }$ due to dissipative phenomena denominate as $T_{d}(t)$. In figure 5 are reported the values of $\Delta T_{\max }$ assessed for each value of $\Delta \sigma / 2$ imposed during fatigue test. In particular, the $\Delta T_{\max }$ values obtained with proposed procedure are represented with blue colour. In the same figure are represented with red colour the not smoothed temperature values. These last were obtained subtracting the environment temperature to the steady state temperature $T_{\max }$ reached during each step [5].

Differences are clearly evident comparing the two series of data. Both are characterized by two different temperature trends correlated at two different behaviours of material. In the first part of fatigue test not damage occurs into material and then the temperature variations that can be observed are negligible. When damage phenomena occur a significant growth of the temperature variation is observed. This behaviour can be approximately represented with a linear function that relates the temperature variations at the level of stress of material.

Filtered data allows to eliminate almost totally the heating effect due to the oleodynamic loading machine and then all the $\Delta T_{\max }$ values are lower than unfiltered ones. As will be showed in the next paragraph, an inaccurate evaluation of all heat sources involved in the measurement of temperature, could compromise the evaluation of fatigue limit of material.

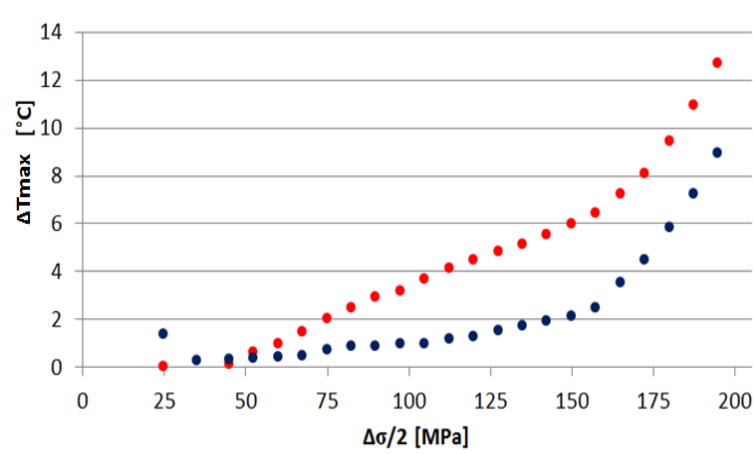

a

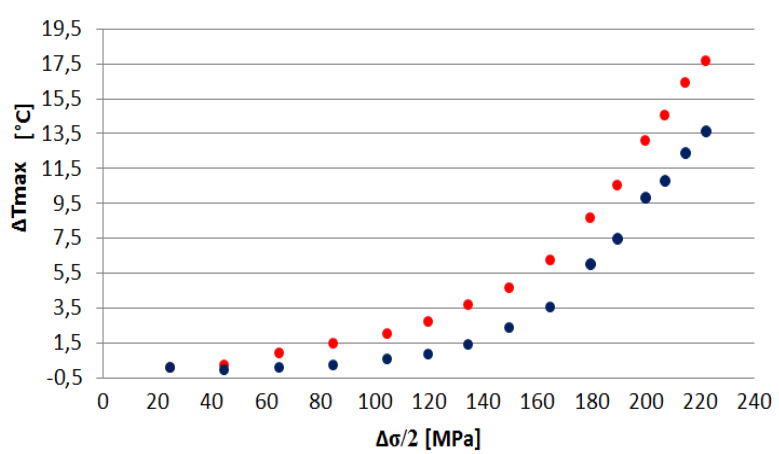

$\boldsymbol{b}$

Fig. 5. Filtered and unfiltered temperature data: a) ASTM A182, b) Virgo 39. 


\subsection{Analysis of phase data}

In figures 6 and 7 are shown the phase maps obtained by thermoelastic data processing described in paragraph 4.2. In particular, the maps are referred to the 3th thermographic sequence acquired around to 16000 cycles.

The phase maps are reported in correspondence of the various load step performed during test. It's clearly evident that the phase variation involves the whole gauge length of the specimen. In particular, the phase signal changes both in positive and negative value. Diaz et. al [22] showed that in presence of a crack growth the phase signal assumes a positive value due to high stress gradients and a negative one due to plastic work. However, future works will be carried out in order to understand the nature of the sign of phase data also in base material. Moreover, in opposite way than temperature, the phase signal changes in some areas of specimen providing a local information about damage phenomena.

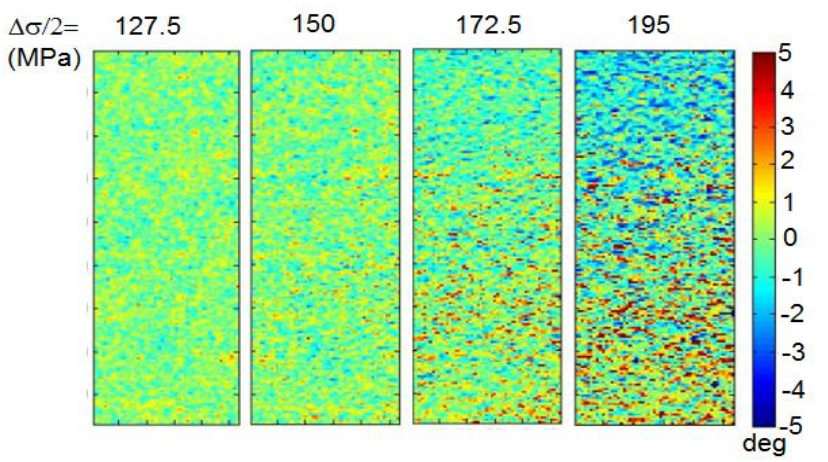

Fig. 6. ASTM A 182 phase images, specimen 1, sub-step 3.

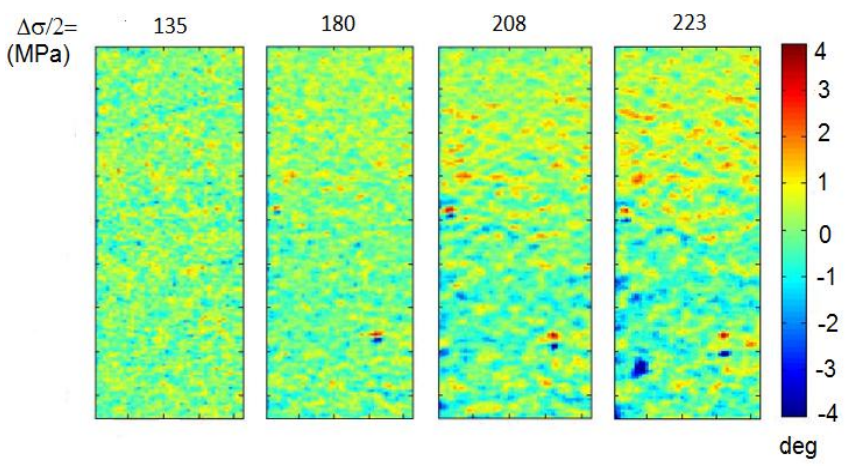

Fig. 7. VIRGO 39 phase images, specimen 3, sub-step 3.

In figure 8 are shown the phase results obtained for the tested materials. The blue line represents the phase data obtained in sub-step 1 at 1000 cycles, the red line the results obtained in sub-step 2 at 8000 cycles and the green line the ones obtained at 16000 cycles. In particular, the comparison among the sub-steps shows that there are not substantially differences in fatigue behaviour of material. The phase signal seems to be related only to stress level and this means it's not necessary to perform the complete step (about 20 minutes) before increasing the load level. So, TPA method can be performed to reduce the test time, because phase signal can be monitored during early cycles.

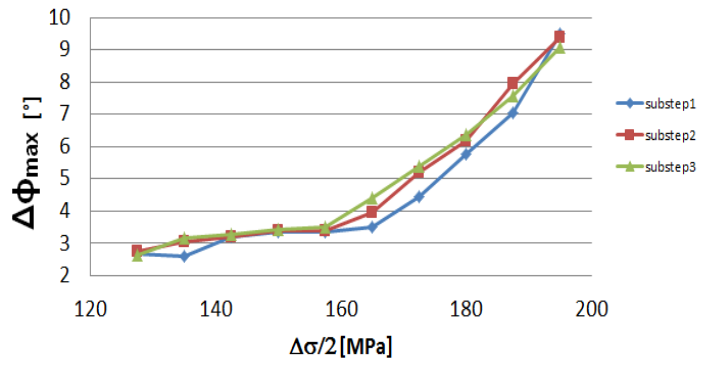

a

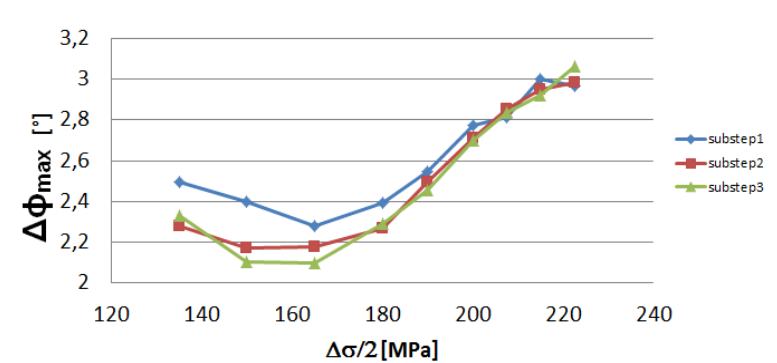

$\boldsymbol{b}$

Fig. 8. Phase signal trends, comparison between the sub-steps results: a)ASTM A182, b)VIRGO 39 


\subsection{Fatigue limit evaluation : temperature data and phase data}

As already found by others author [2-6], the starting point at which the fatigue damage occurs is the break point separating two different behaviour of temperature data. If damage is not presents the temperature variation can be considered negligible. When damage occurs significant temperature variation are observed. Two different behaviour of material are clearly visible and two regression lines can be used to describe the temperature trend. The interaction of two lines allows to obtain fatigue limit.

In figures 9 and 10, the graphical method proposed by Luong [2] is applied to the filtered temperature (a) and phase (b) data, for each material. The differences in number of data points between temperature and phase, are due to the smoothing procedures applied. In fact, as show in previous paragraph, phase data are related to a reference image subtracted during the data processing. In this case the image subtracted is taken at $120 \mathrm{MPa}$, for either Astm a $182 \mathrm{and}$ Virgo 39.

Fatigue limit evaluated on temperature values, is less than the one obtained by phase data. The gap between them, is about 10/20 MPa. The complete results are shown in tables 4 and 5.

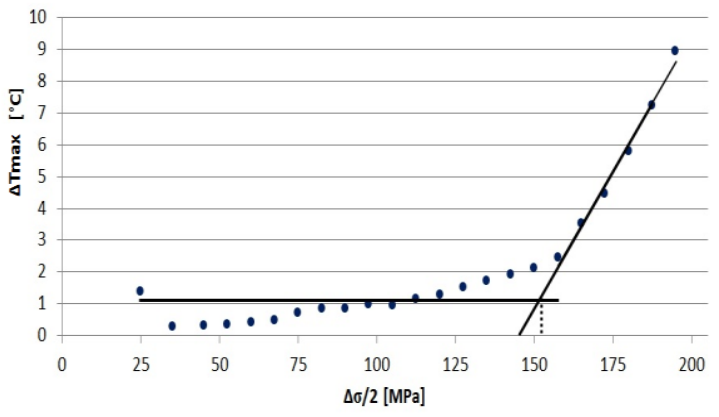

a

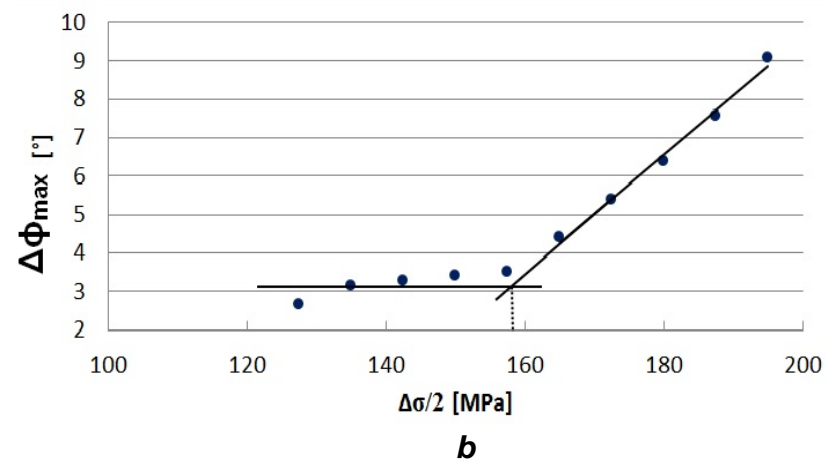

Fig. 9. Fatigue limit evaluation with thermal methods (ASTM A182): a) Temperature data, specimen 1, b) Phase data, specimen 1 , sub-step 3
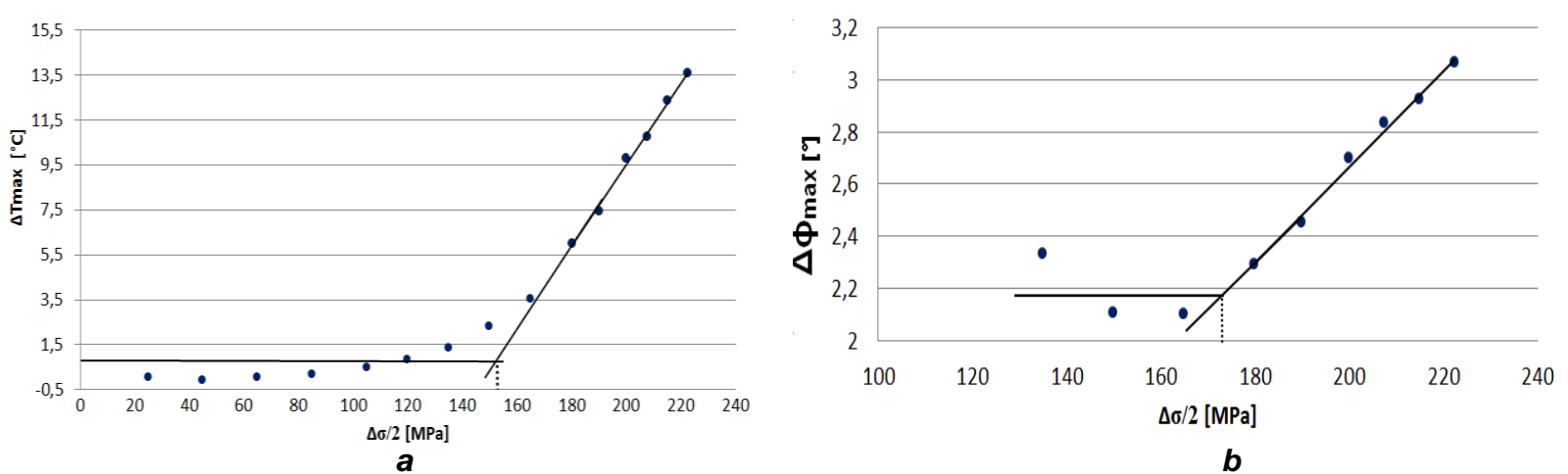

Fig.10. Fatigue limit evaluation with thermal methods (Virgo 39): a) Temperature data, specimen 3, b) Phase data, specimen 3, sub-step 3

\subsection{Comparison between different methods}

According to UNI 3964 [23], a stair-case procedure was carried out on 15 specimens considering $10^{7}$ cycles as limit. The test was performed on ASTM A182 stainless steel, (table 3).

Considering ASTM A 182, the results are represented in table 4. Temperature data underestimates fatigue limit with respect, stair case and phase data.

VIRGO 39 material, wasn't tested with Stair Case method. The results for this material are showed in table 5 . In this case, also, temperature data provides lower values of fatigue limit than phase data. Probably, in presence of low temperatures, the traditional methods used to assess the fatigue limit [2-6] are not reliable, instead the phase analysis provides results very close to "stair-case" method. In fact, martensitic steels are characterized by low deformations and consequent small hysteresis loop during the fatigue loads. This behaviour produces thermal sources and phase values very low respect to others steels such as austenitic [4-6]. 
Unfiltered temperature data, underestimates fatigue limit with respect the temperature data processed with proposed procedure. This means that $\mathrm{n}$ error about $10 \mathrm{MPa}$, can be made if all the heat sources that are involved in fatigue test are not considered.

Table 3. Fatigue limit evaluation: stair-case procedure (ASTM A182)

\begin{tabular}{|c|c|c|c|c|c|c|c|c|c|c|c|c|c|c|c|c|c|c|c|c|c|}
\hline$\Delta \sigma / 2$ & \multicolumn{15}{|c|}{ specimens } & \multicolumn{2}{|c|}{ event } & \multicolumn{4}{|c|}{ parameters } \\
\hline & $\underline{1}$ & $\underline{2}$ & $\underline{3}$ & 4 & $\underline{5}$ & $\underline{6}$ & $\underline{7}$ & $\underline{8}$ & $\underline{\underline{9}}$ & $\underline{10}$ & $\underline{11}$ & $\underline{12}$ & $\underline{13}$ & $\underline{14}$ & $\underline{15}$ & $\mathrm{x}$ & 0 & $\mathrm{i}$ & $n_{i}$ & $i^{*} n_{i}$ & $i^{2} n_{i}$ \\
\hline 180 & & & 1 & & 1 & & & & & & & & & & 1 & 3 & 0 & 2 & 3 & 6 & 12 \\
\hline 170 & & 0 & & 0 & & 1 & & 1 & & 1 & & 1 & & 0 & & 4 & 3 & 1 & 4 & 4 & 4 \\
\hline 160 & 0 & & & & & & 0 & & 0 & & 0 & & 0 & & & 0 & 5 & 0 & 0 & 0 & 0 \\
\hline TOT & & & & & & & & & & & & & & & & 7 & 8 & & 7 & 10 & 16 \\
\hline & & & & & & & & & & & & & & & & & & & $\mathrm{N}$ & A & $B$ \\
\hline
\end{tabular}

Table 4. Fatigue limit: comparison between different methods (ASTM A182).

\begin{tabular}{|c|c|c|c|c|c|c|}
\hline \multirow[t]{2}{*}{ Methods } & \multicolumn{2}{|c|}{ Temperature data } & \multicolumn{3}{|c|}{ Phase data } & Stair Case \\
\hline & $\begin{array}{c}\text { Unfiltered } \\
\text { data } \\
\text { (Mpa) }\end{array}$ & $\begin{array}{l}\text { Filtered data } \\
\text { (Mpa) }\end{array}$ & $\begin{array}{l}\text { Sub } \\
\text { Step } 1 \\
\text { (Mpa) }\end{array}$ & $\begin{array}{c}\text { Sub } \\
\text { Step } 2 \\
\text { (Mpa) }\end{array}$ & $\begin{array}{c}\text { Sub } \\
\text { Step } 3 \\
\text { (Mpa) }\end{array}$ & \\
\hline specimen 1 & 138,28 & 151,16 & 166,96 & 164,50 & 157,98 & \\
\hline specimen 2 & 140,26 & 147,06 & 167,72 & 158,17 & 162,22 & \\
\hline specimen 3 & 142,60 & 149,15 & 170,00 & 160,63 & 157,05 & \\
\hline average & 140,38 & 149,12 & 168,23 & 161,09 & 159,08 & 169,24 \\
\hline St. Dev. & 2,16 & 2,05 & 1,58 & 3,21 & 2,76 & 4,44 \\
\hline
\end{tabular}

Table 5. Fatigue limit: comparison between different methods (VIRGO 39).

\begin{tabular}{|c|c|c|c|c|c|}
\hline Methods & \multicolumn{2}{|c|}{ Temperature data } & \multicolumn{3}{c|}{ Phase data } \\
\hline & $\begin{array}{c}\text { Unfiltered data } \\
\text { (Mpa) }\end{array}$ & $\begin{array}{c}\text { Filtered data } \\
\text { (Mpa) }\end{array}$ & $\begin{array}{c}\text { Sub } \\
\text { Step 1 } \\
\text { (Mpa) }\end{array}$ & $\begin{array}{c}\text { Sub } \\
\text { Step 2 } \\
\text { (Mpa) }\end{array}$ & $\begin{array}{c}\text { Sub } \\
\text { Step 3 } \\
\text { (Mpa) }\end{array}$ \\
\hline specimen 1 & 149,69 & 154,44 & 172,89 & 178,24 & 177,5 \\
\hline specimen 2 & 149,09 & 152,84 & 183,85 & 187,62 & 190,65 \\
\hline specimen 3 & 148,74 & 158,16 & 194,28 & 180,75 & 179,65 \\
\hline average & $\mathbf{1 4 9 , 1 7}$ & $\mathbf{1 5 5 , 1 4}$ & $\mathbf{1 8 3 , 6 7}$ & $\mathbf{1 8 2 , 2 0}$ & $\mathbf{1 8 2 , 6 0}$ \\
\hline St. Dev. & $\mathbf{0 , 2 5}$ & 2,73 & 10,69 & 4,85 & 7,05 \\
\hline
\end{tabular}

\section{Conclusion}

In this work two thermographic techniques were used to study the fatigue behaviour of two martensitic steels: ASTM A182 grade F6NM and Virgo 39. The first method used is based on surface temperature monitoring of specimen during a fatigue test. Temperature is affected by external noise heat sources that could compromise the correct measurement. So, a new procedure of data processing was developed to smooth the temperature data acquired by thermocamera. The comparison between filtered and unfiltered data showed substantial differences in terms of temperature variations measured during the tests. In particular, if the noise heat sources are neglected an error of about $10 \mathrm{MPa}$ can be obtained in the evaluation of fatigue limit with the method present in literature.

The second method used is called TPA and it is based on thermoelastic phase monitoring of specimen during a fatigue test. To obtain the phase signal the thermoelastic stress analysis (TSA) was performed on thermal sequences acquired at regular interval during the fatigue test. In particular it was observed a phase variation from the early cycles of loading when the fatigue limit of material was exceeded. The phase value seems to remain constant until the load conditions are unchanged and it grows once the load increase. These results show as the TPA method could be used to perform a fatigue test more rapid than ones present nowadays in literature.

Significative differences were obtained in terms of fatigue limit using temperature and phase data. In particular for Virgo 39 the phase data provides a fatigue limit very close to Stair Case method.

Moreover, the phase data present different advantages respect to temperature, such as:

- a simpler data processing, 
- $\quad$ can be acquired at any time during the tests while is necessary achieve the steady state temperature to acquire the thermal data,

- $\quad$ are much more robust because less sensitive to environment condition respect to temperature data,

- provides a local information about the state of material.

\section{Acknowledgements}

This work is part of a large-scale research project (PON-SMATI) aimed at identifying innovative steels to turbomachinery used in extreme environmental conditions. The authors would like to thank GE oil \& gas (Nuovo Pignone S.r.I.) for the support and collaboration provided in the experimental tests.

\section{REFERENCES}

[1] Fargione G., Geraci A., La Rosa G., Risitano A., "Rapid determination of the fatigue curve by the thermographic method", International Journal of Fatigue, vol. 24, pp. 11-19, 2001.

[2] Luong M.P., "Fatigue limit evaluation of metals using an infrared thermographic technique", Mech. Mater., vol. 28, pp.155-63, 1998.

[3] Luong M.P., "Infrared thermographic scanning of fatigue in metals", Nuclear Engineering and Design, vol. 158 pp. 363-376, 1995.

[4] La Rosa G., Risitano A., "Thermographic methodology for the rapid determination of the fatigue limit of materials and mechanical components", International Journal of Fatigue, vol. 22, pp. 65-73, 2000.

[5] Morabito A.E., Dattoma V., Galietti U., "Energy-analysis of fatigue damage by thermographic technique", Proc. SPIE, Thermosense XXIV, vol. 4710, 2002.

[6] Curà F., Curti G., Sesana R., "A new iteration method for the thermographic determination of fatigue limit of steels", International Journal of Fatigue, vol. 27, pp. 453-459, 2005.

[7] Chrysochoos A., Louche H., "An infrared image processing to analyse the calorific effects accompanying strain localisation", International Journal of Engineering Science, vol. 38, pp. 1759-1788, 2000.

[8] Giancane S., Dattoma V., Chrysochoos A., Wattrisse B., "Approccio calorimetrico e cinematico alla fatica in una lega di alluminio", XXXV Convegno Nazionale AIAS, Università Politecnica delle Marche, 2006.

[9] Morabito A.E., Chrysochoos A., Dattoma V., Galietti U., "Analysis of heat sources accompanying the fatigue of 2024 T3 aluminium alloys", International Journal of Fatigue, vol. 29, pp. 977-984, (2007).

[10] Krapez J.C., Pacou D., Gardette G., "Lock-in thermography and fatigue limit of metals", Quantitative Infrared Thermography, QIRT, Reims (France), Jul., pp. 18-21, 2000.

[11] Ummenhofer T., Medgenberg J., "On the use of infrared thermography for the analysis of fatigue damage process in welded joints", International Journal of Fatigue, vol. 31, 130-137, 2009.

[12] Crupi V., Guglielmino E., Maestro M., Marinò A., "Fatigue analysis of butt welded AH36 steel joints: Thermographic Method and design S-N curve". Marine Structures, vol. 22, pp. 373-386, 2009.

[13] Palumbo D., Galietti U., "Characterization of steel welded joints by infrared thermographic methods", Quantitative InfraRed Thermography Journal, vol. 11:1, pp. 29-42, 2014.

[14] Casavola C., Galietti U., Modugno D., Pappalettere C., "An application of the differential thermographic technique for welded joints fatigue evaluation”, SPIE Proc., vol. 6250, 2006.

[15] Galietti U., Palumbo D., De Finis R., Ancona F., "Fatigue damage evaluation of martensitic stainless steel by means of thermal methods", XXII Convegno Nazionale IGF, Roma, 1-3 Luglio, pp. 80-90, 2013.

[16] Dulieu-Barton J.M., "Some Introduction to thermoelastic stress analysis", Strain, vol. 35, 35-39, 1999.

[17] Pitarresi G., Patterson E.A., "A review of the general theory of thermoelastic stress analysis", J. Strain Analysis for Engineering Design, vol. 38(5), 405-417, 2003.

[18] Harwood N., Cummings W., "Thermoelastic stress analysis". National Engineering Laboratory, Adam Hilger, Bristol, Philadelphia, New York, 1991.

[19] Wang W.J., Dulieu-Barton J.M., Li Q., "Assessment of Non-Adiabatic Behaviour in Thermoelastic Stress Analysis of Small Scale Components", Experimental Mechanics, vol. 50, 449-461, 2010.

[20] Michael F. McGuire, "Martensitic Stainless Steels", Stainless Steels for Design Engineers, ASM International, pp 123-135, 2008.

[21] ASTM E 466 - Standard Practice for Conducting Force Controlled Constant Amplitude Axial Fatigue Tests of Metallic Materials, 2004.

[22] Diaz F.A., Patterson E.A., Tomlinson R.A., Yates J.R.., "Measuring stress intensity factors during fatigue crack growth using thermoelasticity”, Fatigue Fract. Engng. Mater. Struct, vol. 27, pp. 571-583, 2004.

[23] UNI 3964 - Prove meccaniche dei materiali metallici. Prove di fatica a temperatura ambiente, 2001. 